\title{
INTENSIDADE DE LUZ DE APARELHOS FOTOPOLIMERIZADORES UTILIZADOS NO CURSO DE ODONTOLOGIA DA UNIVERSIDADE ESTADUAL DE PONTA GROSSA
}

\author{
EVALUATION OF CURING LIGHT UNITS AT THE \\ STATE UNIVERSITY OF PONTA GROSSA
}

\author{
Rafaela Lopes Baldi ${ }^{* *}$, Leidi Daiana Teider ${ }^{1}$, Tatiana Moroz Leite ${ }^{1}$, \\ Rosangela Martins ${ }^{1}$, Leyla Antoinette Cotrina Delgado², \\ Stella Kossatz Pereira ${ }^{3}$ \\ 1* Autor para contato: Universidade Estadual de Ponta Grossa - UEPG, Curso de \\ Odontologia, Campus em Uvaranas, Ponta Grossa, PR, Brasil; \\ (42)3027-1471 - 9112-9500; e-mail: rafaela_baldi@yahoo.com.br \\ 2 Universidad Peruana Cayetano Heredia, UPCH, Peru. \\ 3 Universidade Estadual de Ponta Grossa - UEPG, Departamento de Odontologia, \\ Campus em Uvaranas, Ponta Grossa, PR
}

Recebido para publicação em 06/06/2005

Aceito para publicação em 21/07/2005

\section{RESUMO}

O sucesso em um procedimento restaurador realizado pelo cirurgião-dentista está diretamente relacionado com o bom desempenho de seu aparelho fotopolimerizador. Assim sendo, é importante a manutenção do equipamento, tanto de seus componentes como da intensidade de luz por ele emitida, já que o tempo e a freqüência de uso influenciam em seu desempenho. Neste trabalho avaliaramse as condições dos aparelhos fotopolimerizadores do sistema de luz halógena disponíveis no curso de Odontologia da Universidade Estadual de Ponta Grossa. Duas avaliações foram realizadas em períodos de seis meses. Verificaram -se as condições da lâmpada, do filtro, da fibra óptica e também a intensidade de luz emitida. Para a avaliação da intensidade de luz, utilizou-se o radiômetro digital Cure Rite (EFOS). A amostra foi composta por 16 aparelhos dos quais 56,25\% apresentaram-se com intensidade de luz abaixo do valor mínimo preconizado $\left(400 \mathrm{~mW} / \mathrm{cm}^{2}\right)$. Entretanto, durante a segunda avaliação, realizada após 6 meses da primeira amostragem, pôde-se verificar que 69,23\% destes aparelhos apresentaram intensidade de luz abaixo do recomendado pela literatura. Em relação aos componentes do aparelho, as fibras ópticas foram responsáveis pelo maior dano, tanto na primeira como na segunda avaliação. Em contrapartida, as lâmpadas apresentaram-se intactas na primeira avaliação, porém após seis meses, foram registradas fraturas em 7,69\% e opacificação dos bulbos em 15,38\%. Dessa forma, os autores concluíram que os aparelhos fotopolimerizadores do sistema de 
luz halógena utilizados no curso de Odontologia da UEPG necessitam de um programa de manutenção periódica com revisões mensais realizadas por meio de radiômetros manuais.

Palavras-chave: resina composta, intensidade de luz, clínica odontológica

\begin{abstract}
The success in a restorative procedure performed by the professional is directly related to a good performance of the curing light unit. Therefore, the maintenance of the equipment, of its components, and also of the light intensity is important, since the time and frequency affect its performance. In this work, the conditions of the curing lights from the dentistry course of the State University of Ponta Grossa were evaluated. Two evaluations were accomplished in a period of six months. The conditions of the lamp, the filter, the optical fiber and also the light intensity were verified. For the evaluation of the light intensity the digital radiometer Cure Rite (EFOS) was used. The sample consisted of 16 units, of which 56,25\% showed light intensity below the minimum value recomended $\left(400 \mathrm{~mW} / \mathrm{cm}^{2}\right)$. During the second evaluation, measured six months after the first sampling, this index had increased to 69,23\%. Amoug the components of the units, the optical fiber presented the highest degree of damage, both in the first and in the second evaluation. However, the lamps were intact in the first evaluation, but after six months fractures were registered in 7,69\% of the sample and opacifition of the bulbs in 15,38\%. The authors concluded that the curing light units used in the Dentistry course at the University of Ponta Grossa need a program of periodic maintenance consisting of monthly revisions with manual radiometers.
\end{abstract}

Key words: composite resin, light intensity, dental Office

\section{Introdução}

O avanço tecnológico na Odontologia restauradora atravessa uma de suas maiores evoluções em relação à adesão das estruturas dentárias. A resina composta é um material que permite a realização de procedimentos imperceptíveis pela reprodução de características das estruturas dentárias em relação à cor, forma, textura e função, proporcionando restaurações mais naturais. Entretanto, um dos maiores anseios dos profissionais da Odontologia é obter uma restauração estética, mas sem perder a qualidade do material restaurador.

Um grande problema, quando se trata de restaurações mal sucedidas é a inadequada polimerização, que acarreta menor durabilidade destas pela perda das propriedades físico-químicas, sensibilidade pós-operatória, manchamento e infiltração (Pereira et al., 2000; Santos et al.,2003; Vieira et al.,2000).

A maioria das resinas compostas utilizadas em consultórios particulares e clínicas universitárias são ativadas através de luz visível entre 400 e 500nm. Estas iniciam seu processo de polimerização através da absorção de luz por meio de fotoiniciador, a canforoquinona, que uma vez ativada reage com os grupos amina e produz radicais livres (Mazur et al., 2004; Kreidler et al., 2004; Franco e Lopes 2003; Rueggeberg et al.,1994; Vieira et al.,2000). Segundo a literatura, é necessário o tempo de 40 segundos de exposição à luz para polimerizar incrementos de resina composta 
com 2 mm de espessura (Fan et al.,2002; Pereira et al., 2000; Rueggeberg et al.,1994; Santos et al.,2003).

O aparelho de fotopolimerização à base de luz halógena funciona através de um filamento de tungstênio protegido por uma cápsula de quartzo que contém um gás inerte do tipo halógeno. O filamento funciona como uma resistência que é aquecida pela corrente elétrica produzindo radiação eletromagnética em forma de luz visível. A produção exclusiva de luz azul com esta tecnologia não é possível, assim porções não desejadas de espectro devem ser filtradas e o calor também deve ser dissipado com a incorporação de filtros e ventiladores. Embora seja o aparelho mais utilizado, este apresenta algumas desvantagens, como diminuição da intensidade de luz com o passar do tempo. Essa redução de intensidade pode ser explicada pela degradação de seus componentes, como por exemplo escurecimento ou opacificação do bulbo, degeneração do refletor, fraturas ou depósitos de detritos no filtro e perda de reflexão da ponta ativa (Lutz et al., 1992; Miyazaky et al.,1998; Poulos et al., 2000).

Estas considerações são muito importantes, principalmente nas clínicas universitárias, onde os aparelhos fotopolimerizadores são usados com maior freqüência e transportados por diversos lugares da clínica devidos aos múltiplos tratamentos realizados simultaneamente (Poulos et al.,2000).
Para assegurar que o aparelho fotopolimerizador esteja com sua intensidade de luz ideal, é importante realizar a manutenção periódica com o auxílio de radiômetro para garantir adequada polimerização das resinas compostas (Vieira et al., 2000; Poulos et al., 2000; Pereira et al., 2000; Mazur et al., 2004; Kreidler et al., 2004).

Ante o exposto, o objetivo deste trabalho foi avaliar a intensidade de luz e os componentes dos aparelhos fotopolimerizadores do sistema de luz halógena utilizados no curso de Odontologia da Universidade Estadual de Ponta Grossa.

\section{Material e método}

Foram avaliados 16 aparelhos fotopolimerizadores do sistema de luz halógena das clínicas do curso de Odontologia da Universidade Estadual de Ponta Grossa em um intervalo de 6 meses. A primeira avaliação foi realizada para se obter uma leitura inicial e a segunda para podermos comparar os resultados e dessa maneira observar a deterioração dos aparelhos nesse intervalo de tempo. Foi elaborado um questionário para registrar os valores de intensidade de luz e os possíveis danos dos aparelhos (Quadro 1). 
Quadro 1 - Questionário aplicado na pesquisa

\begin{tabular}{|c|c|c|c|}
\hline \multicolumn{4}{|l|}{$\begin{array}{l}\text { APARELHO - } \\
\text { 1. Clinica: } \\
\text { 2. Marca: } \\
\text { 3. Modelo: } \\
\text { 4. Número de Fabricação: } \\
\text { 5. Ano de Fabricação: } \\
\text { 6. Ano de Aquisição: } \\
\text { 7. Métodos de Desinfecção: } \\
\text { 8. Avaliação do Aparelho: }\end{array}$} \\
\hline \multicolumn{2}{|c|}{ DATA } & $1^{\circ}$.Avaliação & $2^{\circ}$.Avaliação \\
\hline \multicolumn{4}{|c|}{ INTENSIDADE DE LUZ $\left(\mathrm{mW} / \mathrm{cm}^{2}\right)$} \\
\hline \multirow{4}{*}{$\begin{array}{l}\text { CONDIÇÕES DA } \\
\text { LÂMPADA }\end{array}$} & BULBO ESCURECIDO & $\begin{array}{l}\text { ( ) SIM } \\
\text { ( ) NÃO }\end{array}$ & $\begin{array}{l}\text { ( ) SIM } \\
\text { ( ) NÃO }\end{array}$ \\
\hline & BULBO OPACIFICADO & $\begin{array}{l}\text { ( ) SIM } \\
\text { ( ) NÃO }\end{array}$ & $\begin{array}{l}\text { ( ) SIM } \\
\text { ( ) NÃO }\end{array}$ \\
\hline & FRATURA & $\begin{array}{l}\text { ( ) SIM } \\
\text { ( ) NÃOO }\end{array}$ & $\begin{array}{l}\text { ( ) SIM } \\
\text { ( ) NÃO }\end{array}$ \\
\hline & $\begin{array}{l}\text { DEGENERAÇÃO } \\
\text { DO REFLETOR }\end{array}$ & $\begin{array}{l}\text { ( ) SIM } \\
\text { ( ) NÃO }\end{array}$ & $\begin{array}{l}\text { ( ) SIM } \\
\text { ( ) NÃO }\end{array}$ \\
\hline \multirow{3}{*}{$\begin{array}{l}\text { CONDIÇÕES DA FIBRA } \\
\text { ÓPTICA }\end{array}$} & FRATURA & $\begin{array}{l}\text { ( ) SIM } \\
\text { ( ) NÃO }\end{array}$ & $\begin{array}{l}\text { ( ) SIM } \\
\text { ( ) NÃO }\end{array}$ \\
\hline & DETRITOS & $\begin{array}{l}\text { ( ) SIM } \\
\text { ( ) NÃO }\end{array}$ & $\begin{array}{l}\text { ( ) SIM } \\
\text { ( ) NÃO }\end{array}$ \\
\hline & $\begin{array}{l}\text { CAPACIDADE DE } \\
\text { REFLEXÃO }\end{array}$ & $\begin{array}{l}\text { ( ) SIM } \\
\text { ( ) NÃO }\end{array}$ & $\begin{array}{l}\text { ( ) SIM } \\
\text { ( ) NÃO }\end{array}$ \\
\hline \multirow{3}{*}{ CONDIÇÕES DO FILTRO } & DETRITOS & $\begin{array}{l}\text { ( ) SIM } \\
\text { ( ) NÃO }\end{array}$ & $\begin{array}{l}\text { ( ) SIM } \\
\text { ( ) NÃO }\end{array}$ \\
\hline & FRATURA & $\begin{array}{l}\text { ( ) SIM } \\
\text { ( ) NÃO }\end{array}$ & $\begin{array}{l}\text { ( ) SIM } \\
\text { ( ) NÃO }\end{array}$ \\
\hline & OUTROS & $\begin{array}{l}\text { ( ) SIM } \\
\text { ( ) NÃO }\end{array}$ & $\begin{array}{l}\text { ( ) SIM } \\
\text { ( ) NÃO }\end{array}$ \\
\hline
\end{tabular}

Para a aferição da intensidade de luz foi utilizado o radiômetro digital Cure Rite (EFOS) segundo o protocolo estabelecido a partir de estudos registrados na literatura (Pereira et al.,1996; Pereira et al.,2003; Poulos et al.,2000; Vieira et al.,2000). O aparelho fotopolimerizador foi acionado por 60 segundos para aquecer a lâmpada halógena. Em seguida foram realizadas 3 leituras durante 10 segundos, para assegurar a repetição da leitura e um intervalo de dez segundos foi deixado entre cada medição. O resultado foi a média aritmética expressa em $\mathrm{mW} / \mathrm{cm}^{2}$.

Todos os aparelhos foram avaliados no mesmo dispensário e na mesma tomada para que não houvesse diferença na voltagem elétrica.

Em relação ao estudo dos componentes internos dos aparelhos fotopolimerizadores, foi necessária a presença de um técnico para se fazer uma melhor análise. No momento da avaliação da lâmpada analisou-se a presença de fraturas, detritos, escurecimento e opacificação do bulbo. Na fibra óptica analisaram-se resíduos, fraturas e se havia capacidade de reflexão. Para isto a fibra óptica foi colocada sobre um texto impresso e quando as palavras apresentavam-se nítidas na extremidade da fibra, comprovava-se o bom desempenho da fibra óptica. E finalmente, no filtro analisaramse fraturas, detritos e manchas.

Sempre que o aparelho apresentava algum tipo de dano o fato foi comunicado ao técnico para que fossem tomadas as devidas providências, como troca da lâmpada, do filtro e da fibra óptica. Também foi aconselhado que as pontas de fibras ópticas fossem limpas com gaze embebida em álcool 70\% ao final de cada aula. 


\section{Resultados e discussão}

A qualidade da restauração é determinada pelo material restaurador a ser utilizado, técnica correta de inserção da resina composta, tipo de aparelho fotopolimerizador, assim como a polimerização adequada do material (Mazur et al., 2004).

Neste estudo os 16 aparelhos testados apresentaram valores de intensidade de luz entre $118 \mathrm{~mW} /$ $\mathrm{cm}^{2}$ e $573 \mathrm{~mW} / \mathrm{cm}^{2}$ (Gráfico 1). Na primeira avaliação $56,25 \%$ dos aparelhos apresentaram intensidade de luz abaixo do valor preconizado pela literatura - $400 \mathrm{~mW} /$ $\mathrm{cm}^{2}$ (Poulos et al.,1992; Pereira et al.,2000). Já na segunda avaliação este percentual aumentou para $69,23 \%$. Isto porque na segunda avaliação o número de aparelhos da amostra foi reduzido para treze, pois os três restantes encontravam-se em manutenção.

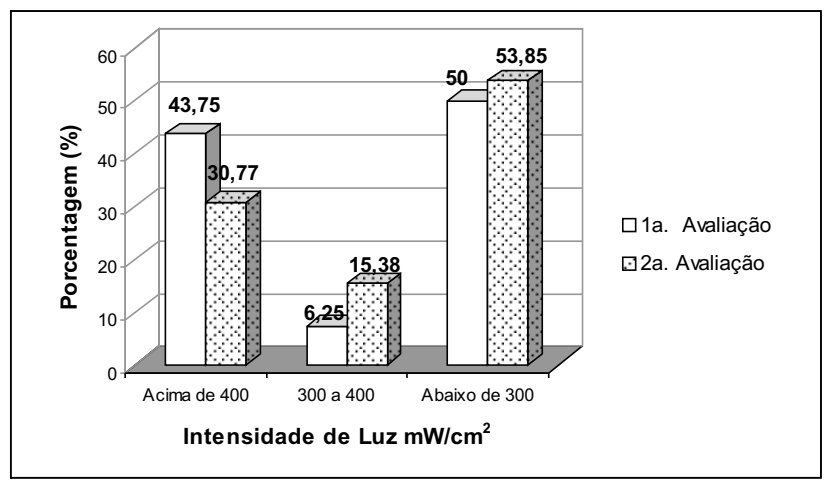

Gráfico 1 - Valor da intensidade de luz segundo o tempo

Estes resultados são concordantes com os encontrados por Poulos et al., (2002) que afirmaram que a degradação sofrida pelos componentes do aparelho fotopolimerizador em um curto intervalo de tempo gera redução na intensidade de luz.

Barghi et al., (1994) avaliaram 209 aparelhos fotopolimerizadores de consultórios particulares e constataram que $30 \%$ eram inadequados para promover a adequada polimerização, pois apresentavam intensidade de luz abaixo de $200 \mathrm{~mW} / \mathrm{cm}^{2}$. E $15.8 \%$ desses aparelhos necessitavam de aumento no tempo de exposição à luz para promover uma adequada polimerização.

Pereira et al., (1996) avaliaram 120 aparelhos de consultórios particulares, $60 \%$ dos quais apre- sentavam valores de intensidade de luz entre 100 e $200 \mathrm{~mW} / \mathrm{cm}^{2}$. Segundo os autores, esses valores não são suficientes para promover uma polimerização adequada. Esses autores também observaram que a maioria dos profissionais desconheciam o valor de emissão de luz de seus equipamentos.

Como esses resultados foram obtidos em consultórios particulares, percebe-se que a falta de controle periódico na manutenção dos aparelhos não se restringe a clínicas universitárias. Muitos profissionais não fazem regularmente a aferição da intensidade de luz de seus aparelhos, permitindo que estes atuem de forma inadequada. Os resultados deste estudo assim como os resultados de Mazur et al. (2004) mostraram a necessidade do uso de radiômetros para a verificação da intensidade de luz, tanto nas universidades como nos consultórios particulares.

Quanto aos valores de intensidade de luz, recomenda-se que aparelhos com intensidade inferior a $200 \mathrm{~mW} / \mathrm{cm}^{2}$ sejam encaminhados para a manutenção, pois a resina composta não será polimerizada adequadamente. Aparelhos com intensidade de luz entre 201 a 399mW/cm² são aceitáveis, porém requerem um tempo adicional de polimerização (Kreidler et al., 2004). Fan et al., (2002) afirmaram que intensidades de luz de $300 \mathrm{~mW} / \mathrm{cm}^{2}$ são suficientes quando o tempo de exposição apropriado é utilizado. E aparelhos com intensidade de luz superior a $400 \mathrm{~mW} / \mathrm{cm}^{2}$ podem ser utilizados com o tempo de exposição de 40 segundos para incrementos de $2 \mathrm{~mm}$ de resina composta (Long et al.,1992; Lutz et al.,1992; Vieira et al.,2000; Kreidler et al., 2004).

No que diz respeito aos componentes do aparelho, édefundamental importância sua manutençãoperiódica, visto que sua degradação resultará na perda de desempenho do aparelho, pois existe uma importante relação entre intensidade de luz, freqüência e tempo de uso dos aparelhos fotopolimerizadores (Kreidler et al., 2004).

A porcentagem de danos encontrados nos componentes dos aparelhos fotopolimerizadores (lâmpada, filtro e fibra óptica) podem ser observados no Gráfico 2. Segundo estes resultados, o componente que apresentou maior relevância foi a fibra óptica, com 100\% na primeira avaliação e 69,23\% na segunda avaliação. Esta redução ocorreu porque nesse intervalo de tempo alguns aparelhos foram para a manutenção, e foram substituídas as fibras fraturadas. Na primeira avaliação 
as responsáveis pela limpeza dos aparelhos foram orientadas para limpar a ponta da fibra óptica friccionando-a com gaze embebida de álcool $70 \%$. O filtro apresentou 43,75\% de danos na primeira avaliação e 46,15\% na segunda avaliação.

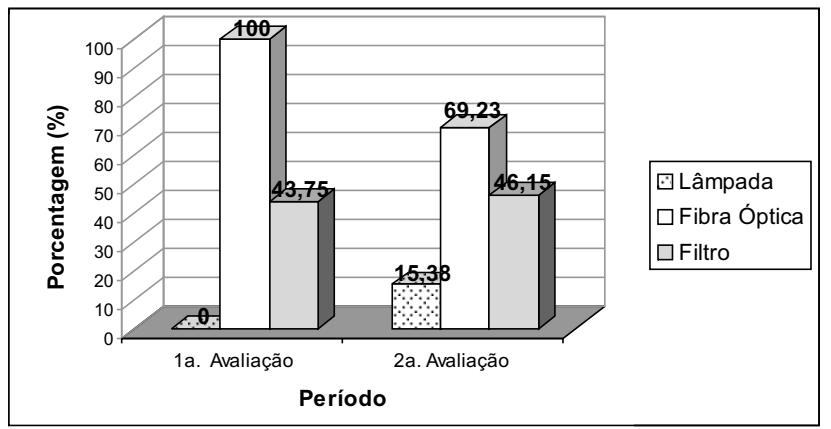

Gráfico 2 - Porcentagem de danos encontrados nos componentes dos aparelhos fotopolimerizadores do sistema de luz halógena.

Em relação à lâmpada halógena, sua vida útil é de 40 horas, ou seja, de 6 a 12 meses dependendo da freqüência e dos ciclos de "acende-apaga” a que é submetida (Kreidler et al., 2004; Vieira et al., 2000). Neste trabalho, esse componente foi o menos significativo, pois como o aparelho é utilizado por muitos acadêmicos do curso de Odontologia, há um maior desgaste da lâmpada, requerendo uma manutenção periódica. Contudo, na segunda avaliação algumas lâmpadas apresentaramse opacificadas e com fraturas no bulbo. Essas lâmpadas eram provavelmente mais antigas, portanto já apresentavam uma redução na intensidade de luz (Gráfico 3). Segundo Miyazaki et al., (1999) a substituição da lâmpada resulta em aumento na intensidade de luz de 36\%. Por esse motivo, tanto profissionais como acadêmicos devem estar sempre atentos à condição em que se encontra a lâmpada de seu aparelho fotopolimerizador.

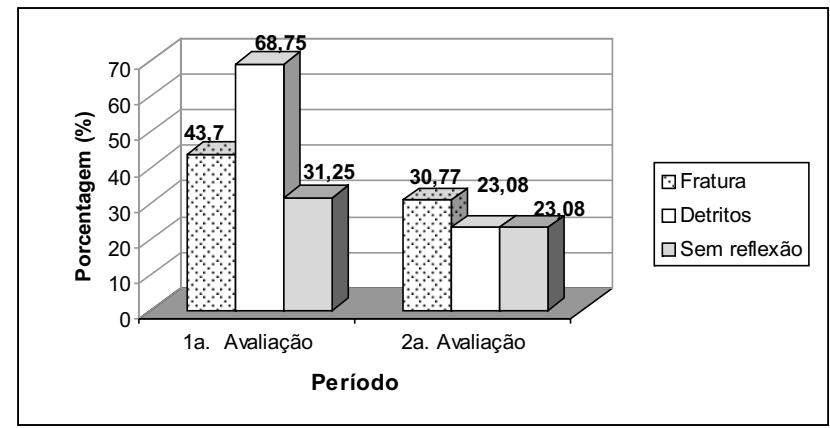

Gráfico 3 - Porcentagem de danos encontrados nas lâmpadas halógenas.

Em relação à fibra óptica, os resultados mostraram que na primeira avaliação 68,75\% dos aparelhos apresentaram detritos, já na segunda avaliação 30,77\% dos aparelhos apresentavam fibras ópticas fraturadas (Gráfico 4). Fraturas e detritos podem provocar uma redução de 46,2\% na emissão de intensidade de luz (Miyazaki et al.,1999).

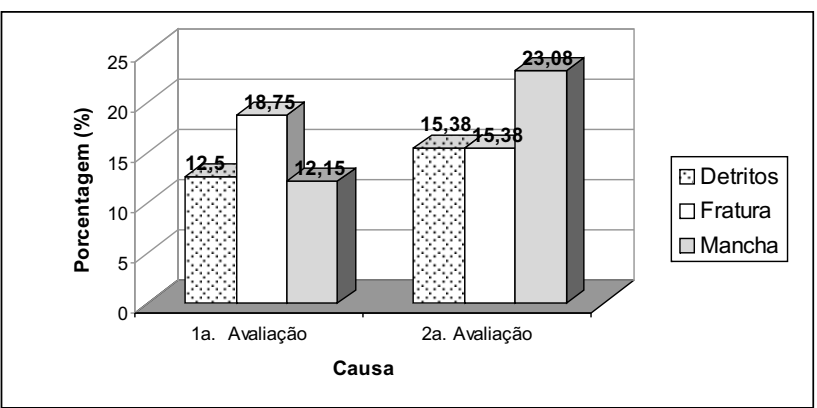

Gráfico 4 - Porcentagem de danos encontrados nas fibras ópticas

De acordo com os resultados da primeira avaliação, $18,75 \%$ dos filtros apresentaram fraturas. $\mathrm{Na}$ 
segunda avaliação 23,08\% dos aparelhos apresentaram manchas na superfície de seus filtros (Gráfico 5). Segundo Lutz et al. (1992) essas manchas podem ser originadas pela queima de sujeira ou por superaquecimento e podem gerar perda de rendimento de $90 \%$. O filtro é o responsável pela emissão do comprimento de onda da luz ideal para a polimerização, que deve ser entre 400 a 500nm. Segundo Bargui et al. (1994), 10\% dos aparelhos avaliados em seu trabalho apresentaram filtros fraturados. Miyazaki et al., (1999) afirmaram que a substituição da lâmpada, da fibra óptica e do filtro aumentaram em $322,7 \%$ o valor da inten-sidade de luz.

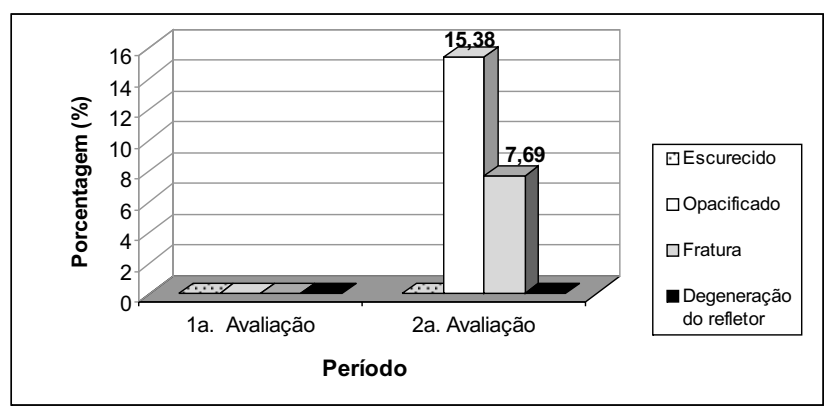

Gráfico 5 - Porcentagem de danos encontrados no filtro.

A biossegurança é fundamental para a proteção de pacientes e de profissionais. Por isso é necessária uma rigorosa desinfecção dos aparelhos fotopolimerizadores, pois estes podem ser responsáveis por infecções cruzadas.

Neste estudo constatou-se que os aparelhos são submetidos à limpeza com álcool $70 \%$, no final do dia, pelas funcionárias responsáveis pela clínica. Essa desinfecção também é de responsabilidade do acadêmico sempre que ocorre a troca de pacientes. Outra maneira de se evitar uma infecção cruzada é utilizando-se uma película de microfilme (Rolopac ${ }^{\circledR}$ ) em todo o aparelho fotopolimerizador, inclusive em sua ponteira.

Segundo Pereira et al. (2003) o método de desinfecção com álcool $70 \%$ foi eleito por $80 \%$ de seus entrevistados. Lutz et al. (1992) afirmaram em seu estudo que soluções desinfetantes podem causar danos irreversíveis no aparelho, principalmente soluções à base de glutaraldeído. Uma maneira de limpar a ponta de fibra óptica e não danificá-la é através da sua imersão em água para que restos de resina composta e selante absorvam água e sejam facilmente removidos com uma espátula de madeira. Entretanto Kleider et al. (2004) afirmaram em seu trabalho que $31 \%$ dos aparelhos eram limpos com desinfetante contendo glutaraldeído e este não demonstrou interferência na intensidade de luz emitida pelos aparelhos fotopolimerizadores. Contudo o uso de álcool 70\% é consagrado na literatura por não causar nenhum tipo de dano ao aparelho fotopolimerizador.

A partir dos resultados encontrados neste estudo, fica evidente a importância de um correto programa de manutenção dos aparelhos fotopolimerizadores do sistema de luz halógena com revisões periódicas de seus componentes - fibra óptica, lâmpada e filtro - e aferições regulares da intensidade de luz com radiômetros manuais.

\section{Conclusão}

Neste trabalho foram realizadas duas avaliações com um intervalo de seis meses para que se pudesse comparar os resultados e assim avaliar a deterioração dos aparelhos fotopolimerizadores das clínicas de Odontologia da UEPG. A partir dos resultados obtidos ao término da segunda avaliação, pôde-se concluir que:

- 69,23\% dos aparelhos avaliados apresentaram intensidade de luz abaixo do preconizado pela literatura;

- As fibras ópticas apresentaram danos em $69,23 \%$ dos aparelhos, os filtros em $46,15 \%$ e as lâmpadas em 15,38\%;

- Os aparelhos fotopolimerizadores utilizados no curso de Odontologia da UEPG necessitam de um programa de manutenção periódica e revisões mensais realizadas por meio de radiômetros manuais.

\section{REFERÊNCIAS}

1. BARGHI, N.; BERRY, T.; HATTON, C. Evaluating intensity output of curing lights in private dental offices. J Am Dent Assoc, v.125, p.992-996. 1994.

2. FAN, P.L.; SCHUMACHER, R.M.; AZZOLIN, K.; GEARY, R.; EICHMILLER, F.C. Intensidade de luz polimerizadora e profundidade de polimerização de resinas compostas testadas de acordo com os padrões internacionais. JADA - Brasil, v.5, p.199-204, 2002. 
3. FRANCO, E.B.; LOPES, L.G.; WANG, L. Resina condensável, uma opção para dentes posteriores: relato de um caso clínico. J Bras Clin Odontol Integr, v.4, p.75-79, 2000.

4. KREIDLER, M.A. de M. LIMA, D.M.; RASTELLI, A.N. de S.; ANDRADE, M.F. Avaliação da intensidade de luz de aparelhos fotopolimerizadores. J Bras Clin Odontol Integr, v. 8, p. 249-53, 2004.

5. LONG, W.R.; CURBOX, S.C. In office survey of curing light maintenance. J Indiana Dent Assoc, v.71, n.3, p.9-11, 1992.

6. LUTZ, F.; KREJCI, I.; FRISCHKNECHT, A. Lichtpolymerisations gerate. Schweiz Monatsschr Zahmed, v.102, p.565-572, 1992.

7. MAZUR R.F.; IGNACIO, S.A.; FERRONATO, T.; KOERICH, G.; VIEIRA, S. Avaliação da qualidade dos aparelhos fotopolimerizadores da clínica odontológica da PUC-PR. Rev Ibero-americana de Odontologia Estética \& Dentística, v. 3, p. 160-8, 2004.

8. MIYAZAKI, M.; HATORRI, T.; ICHIISH, Y.; KONDO, M.; ONOSE, H.; MOORE, B.K. Evaluation of curing light used in private dental offices. Operative Dentistry, v.23, n.2,p.50$54,1998$.

9. PEREIRA, S.K.; PORTO, C.L. de A.; MANDARINO, F.; RODRIGUES. Análise de aparelhos fotopolimerizadores aspectos clínicos relacionados a manutenção, eficiência e emissão da intensidade de luz. Rev Gaúcha Odont, v.44, n.3, p.143-145, 1996.

10. PEREIRA, S.K.; PORTO, C. L. de A.; MANDARINO, F.; RODRIGUES, A.L. JR. Intensidade de luz e profundidade de polimerização de aparelhos fotopolimerizadores. Rev Assoc Paul Cir Dent, v.51, n.3, p.257-260, 1997.

11. PEREIRA, S.K. Resina composta fotopolimerizável. Avaliação da dureza superficial em função de: cor, tempo de exposição, intensidade de luz e profundidade do material. Araraquara, 1999. 216p. Tese (Doutorado em Dentística Restauradora). Faculdade de Odontologia, Universidade Estadual Paulista.

12. PEREIRA, S.K.; PORTO, C.L.A.; MENDES, A.J.D. Avaliação da dureza superficial de uma resina composta híbrida em função da cor, tempo de exposição, intensidade de luz e profundidade do material. J Bras Clin Estet Odont, Curitiba, v.4, n.23, p.6367, 2000.

13. PEREIRA, S.K.; PORTO, C.L.A.; MENDES, A.J.D. Efeitos de diferentes sistemas de fotopolimerização na dureza superficial da resina composta. J Bras Clin Estet Odont, Curitiba, v.5, n.26, p.156-161, 2001.

14. PEREIRA, S.K.; PASCOTTO, R.C.; CARNEIRO, F.P. Avaliação dos aparelhos fotopolimerizadores utilizados em clínicas odontológicas. J Bras Dent Estet, Curitiba, v.2, n.5, p.29-35, jan./mar. 2003.

15. POULOS, J. G; STYNER, D. Lâmpada de polimerização: alterações no rendimento da intensidade de luz com o tempo de trabalho. Jornal de Clínica em Odontologia, artigo n.6, p.4753,2000

16. RUEGGEBERG F.A.; CRAIG R.G. Correlation of parameters used to estimate monomer conversion in a light-cured composite. J Dent Res, v.19, p.26-32, 1994.

17. SANTOS, A.P.D.; MONTANDON, A.A.B.; CHÁVEZ, O.F.M. Avaliaçã0o dos fotopolimerizadores utilizados por clínicos gerais de Araraquara - SP. 20a reunião da SBPqO, 2003 (Abstract Ia121).

18. VIEIRA, G.F.; ERHARDT, A.E.; SHROEDER, L.F. Intensidade de luz de aparelhos fotopolimerizadores utilizados em consultórios particulares. J Bras Clin Estet Odont, v.4, n.22, p.41-44, 2000. 